\title{
Microscopic structure of Fe-Ni and Fe-Ni-S molten alloys of geophysical interest
}

\author{
G. J. Cuello \\ Institut Laue Langevin, 6 rue Jules Horowitz, BP 156, F-38042 Grenoble Cedex 9, France \\ R. Fernández-Perea and C. Cabrillo \\ Instituto de Estructura de la Materia, C.S.I.C., Serrano 123, E-28006 Madrid, Spain \\ F. J. Bermejo* \\ Department of Electricity and Electronics, University of the Basque Country, Box 644, 48080 Bilbao, Spain
}

G. Román-Ross

Laboratoire de Géophysique Interne et Tectonophysique, Maison de Géosciences, UJF-CNRS, BP 53 F-38041 Grenoble Cedex 9 , France

(Received 17 July 2003; revised manuscript received 1 October 2003; published 12 March 2004)

\begin{abstract}
The static $S(Q)$ liquid structure factors for binary $\mathrm{Fe}_{x} \mathrm{Ni}_{1-x}, x=0.90,0.85$, and ternary $\mathrm{Fe}_{0.85} \mathrm{Ni}_{0.05} \mathrm{~S}_{0.15}$ molten alloys are investigated by means of the concurrent use of neutron diffraction and reverse Monte Carlo simulations. The measured $G(r)$ radial distributions reveal atomic orderings varying from that present in the $\mathrm{Fe}_{0.90} \mathrm{Ni}_{0.10}$ alloy, reminiscent of the ideal bcc structure of solid $\mathrm{Fe}$, to a far more open structure found in $\mathrm{Fe}_{0.85} \mathrm{Ni}_{0.05} \mathrm{~S}_{0.15}$. From data at hand no clear evidence of the clustering of sulfur within the Fe-Ni matrix is found. In contrast, the addition of sulfur leads to significant changes in structural and some dynamical properties that can be inferred from knowledge of the static structure factors. Such dynamical changes seem to arise as a result of a strong decrease of the elastic moduli of the alloys resulting from interactions with a light element rather than from a density effect.
\end{abstract}

DOI: 10.1103/PhysRevB.69.094201

PACS number(s): 61.25.Mv, 62.60.+v, 96.35.-j

\section{INTRODUCTION}

Our present understanding of the nature of the inner core of planets identifies light alloying elements as necessary ingredients to explain a wealth of seismic data. ${ }^{1}$ Liquid $\mathrm{Fe}$ and $\mathrm{Fe}-\mathrm{Ni}$ alloys as well as those of the same mixture with the addition of elements such as $\mathrm{O}, \mathrm{Si}$ or $\mathrm{S}$ are being extensively investigated, especially at high temperatures and pressures, ${ }^{2}$ trying to emulate the conditions of planetary interiors. In fact, the available evidence from seismological sources as well as relevant planetological data measured by spacecraft probes $^{3}$ point towards the metallic liquid nature of the outer cores of several planetary bodies.

Testing several Earth models against shock-wave pressure-density and compressibility data at high pressure, Birch $^{1}$ pointed out that the outer liquid core of the Earth was less dense (by 10\%) than bulk iron within the 133-330 GPa pressure range. At pressures of 330-364 GPa, a density close to that of pure iron matches models of the Earth. ${ }^{4}$ Several light elements (e.g., O, S, Si, and C) have been suggested as possible alloying elements, which, upon dissolving in liquid iron in the outer core, can account for its lower density. For reasons easy to gauge, special attention was paid to the characterization of thermal and mechanical properties of mixtures such as the iron-nickel sulfide alloy, since accurate knowledge of them is required as input for thermomechanical models of planetary cores. This kind of result is relevant also to studies of solar system formation and comparative planetology, including that of Earth. Io, the innermost Galilean satellite of Jupiter, and Earth, Ganymede, and Mars seem to be the only bodies in the solar system for which a metallic core has been directly detected either by means of seismic waves (Earth) or by measurements of the gravitational field (Io, Ganymede, Mars). 5,6,3 Given their relative locations in the solar system, this remarkable similarity must be accounted for in theories of solar system origin and evolution.

What led us to focus our interest these molten alloys was a relatively recent paper $^{7}$ that has reported a highly anomalous behavior of the ultrasonic sound velocity $c_{v}(T)$ and attenuation $a(T)$ in the mixture $85 \% \mathrm{Fe}-5 \% \mathrm{Ni}-10 \% \mathrm{~S}$ for temperatures above melting $\left(T_{\mathrm{m}}=1650 \mathrm{~K}\right)$ up to $2000 \mathrm{~K}$ under its saturated vapor pressure. In fact and contrary to data for the pure liquid-metal components, ${ }^{8}$ the acoustic velocity for such a sample increases with temperature with a rate of $\mathrm{d} c / \mathrm{d} T=0.625 \mathrm{~m} \mathrm{~s}^{-1} \mathrm{~K}^{-1}$ at $2000 \mathrm{~K}$. Such a figure is to be compared with the temperature dependence of the sound velocity in molten iron, nickel, and sulfur, which show rates of decrease of $1,0.35$, and $1.26 \mathrm{~m} \mathrm{~s}^{-1} \mathrm{~K}^{-1}$ (Ref. 8) respectively. On the other hand, the quantity actually reported in Ref. 7, which is inversely proportional to the attenuation per wavelength, decreases with increasing $T$ at a rate which can be estimated as $0.033 \mathrm{~K}^{-1}$. Hydrodynamically, the main contributions to acoustic attenuation are the longitudinal viscosity and a heat-conduction term. The former quantity comprises bulk and shear viscosities. Again, the temperature dependence of the viscosity of the pure metals decreases with increasing temperature. ${ }^{8}$

All sources of possible artifacts in Ref. 7 (segregation, loss of sulfur from sample) seem to have been taken care off, and on such a basis the existence of some form of microscopic aggregation is postulated as a plausible explanation for the observed acoustic anomaly. ${ }^{7}$ In fact, some electronic properties of the pure liquid components are shown to 
be deeply affected by the presence of elements such as $\mathrm{Si}$ or $\mathrm{S},{ }^{9}$ something which is expected to lead to substantial changes of structure. If such "molecular" units exist in the liquid as a consequence of some sort of S-induced rearrangements, then the breakup of these units with increasing temperature will explain the increase in sound velocity (greater packing efficiency of the liquid) as well as the anomalous behavior of the attenuation.

In addition, the advent of first-principles molecular dynamics simulations of molten Fe (Refs. 10 and 11) and FeS (Refs. 12) mimicking the conditions of the Earth interior provide us with a valuable tool to gain access to quantities not easily amenable to experiment. Particular attention merit some recent reports ${ }^{12}$ on the effects brought forward by light elements such as sulfur on the microscopic structure of molten iron.

Here we report on a neutron diffraction study of the liquid mixtures referred to above within the temperature range $1700-2000 \mathrm{~K}$, aiming to detect significant differences of structure at scales of a few tens of angstroms. The structure of the liquid-metallic elements ${ }^{13}$ as well as that of molten sulfur ${ }^{14}$ has been determined with substantial accuracy. Two compositions of the $\mathrm{Fe}_{x} \mathrm{Ni}_{1-x}, x=0.9$ and $x=0.85$, binary alloys are studied to check whether significant differences arise due to composition and these are compared to a $\mathrm{Fe}_{0.85} \mathrm{Ni}_{0.05} \mathrm{~S}_{0.10}$ alloy. This will enable us to distinguish whether significant positional correlations develop with the addition of a light component or, on the contrary, the structure of the ternary alloy conforms to that of a random mixture of the three components.

\section{EXPERIMENTAL DETAILS}

Three samples with compositions $\mathrm{Fe}_{0.90} \mathrm{Ni}_{0.10}$, $\mathrm{Fe}_{0.85} \mathrm{Ni}_{0.15}$, and $\mathrm{Fe}_{0.85} \mathrm{Ni}_{0.05} \mathrm{~S}_{0.10}$ were prepared by Metal Laboratory, Centro Atómico Bariloche, Argentina. The alloys were prepared from high-purity $\mathrm{Fe}, \mathrm{Ni}$, and $\mathrm{FeS}$ in an arc furnace, on a water-cooled copper hearth, using nonconsumable electrodes under a 350-Torr Ar atmosphere. Each alloy was melted at least six times in order to ensure sample homogeneity.

The neutron diffraction experiments were performed using the D4C instrument ${ }^{15}$ at the Institut Laue-Langevin (Grenoble, France) with an incident neutron wavelength of $0.7117(1) \AA$ A. This wavelength and the corresponding zeroangle correction $0.1834(2)^{\circ}$ were determined by means of an independent measurement using a $\mathrm{Ni}$ powder sample as standard. The samples were held in cylindric sapphire containers of $10.0 \mathrm{~mm}$ internal diameter and $1.0 \mathrm{~mm}$ wall thickness, placed in a high-temperature tungsten furnace, which in time was placed in the evacuated instrument belljar. This furnace consists of two concentric tungsten surfaces: a heating element of $4.6 \mathrm{~cm}$ diameter and $0.06 \mathrm{~mm}$ thickness and an external wall of $121 \mathrm{~mm}$ internal diameter and $3.6 \mathrm{~mm}$ thickness. ${ }^{16}$

Diffraction patterns were taken for the samples in their container and the empty container, in the range of $1.5^{\circ}-140^{\circ}$. The empty container spectrum at high temperature $(1823 \mathrm{~K})$ was measured for each sample after rotating

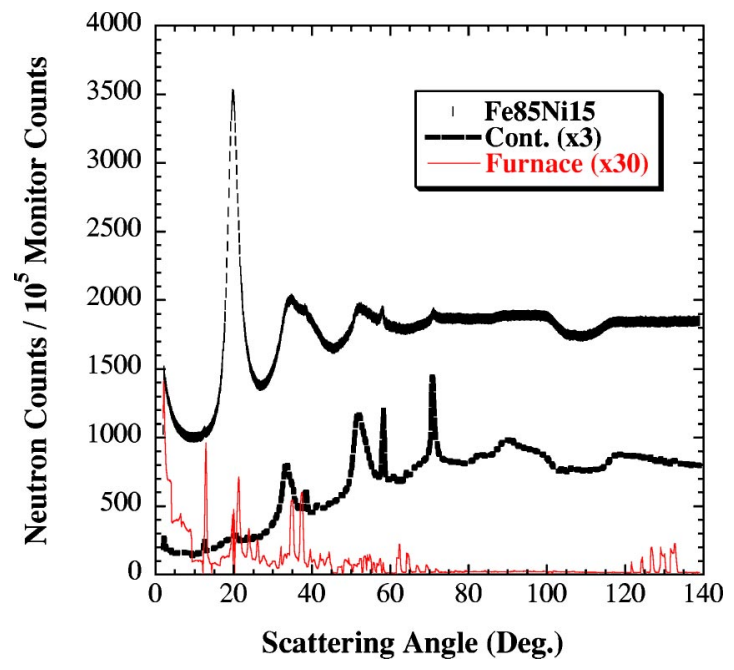

FIG. 1. Measured raw intensities at $1823 \mathrm{~K}$ for a sample of $\mathrm{Fe}_{0.85} \mathrm{Ni}_{0.15}$, the empty sample container and the empty oven. Notice the magnifications employed to display the latter two.

the container around its axis to determine the position for which fewer Bragg peaks fall in the spectrum. This is possible because each container is built up from a sapphire single crystal in such a way that none of its principal axes was parallel to the cylinder axes. Once the container was cooled, it was filled with the sample and heated again up to $1823 \mathrm{~K}$ until complete melting was achieved. A diffraction pattern at room temperature was also taken for a vanadium rod of dimensions comparable to the sample for normalization purposes. The intensity for the empty furnace and a cadmium neutron-absorbing rod of similar diameter to the sample were also measured to account for the effect of sample self-shielding on the background count rate at small scattering angles. ${ }^{17}$ Each complete diffraction pattern was built up from the intensities measured for the different detector groups. These intensities were saved at regular intervals and no deviation between them was observed, apart from the expected statistical variations, which verified the diffractometer and sample stability. ${ }^{18}$ A sample of raw data is shown in Fig. 1. Notice that while there is in principle data extending up to $140^{\circ}$ a strong dip is seen in the curves labeled as container and sample plus container centered at about $110^{\circ}$. This arises from a tungsten rod that supports the heating element. Since it is off centered with respect to the sample position, an accurate analytical correction of this effect is hard to pursue. While under similar circumstances data smoothing and interpolation to remove such parasitic scattering are common practices, we have chosen to limit our angular range to $95^{\circ}$. This leads to some reduction in realspace resolution but prevents the introduction of possible artifacts brought forward by the employed data filtering procedures.

The measured distributions of neutron counts versus scattering angle were first corrected for absorption and multiple scattering effects by means of the CORRECT code. ${ }^{19}$ The procedure required some previous knowledge of the densities of the molten alloys. To do so the corresponding molar compositions were calculated (Table I), and an initial 
TABLE I. Composition of each sample and their corresponding coherent scattering lengths and densities.

\begin{tabular}{cccc}
\hline \hline Composition by weight & Molar composition & $b_{\text {coh }}^{2}(\mathrm{~b} / 4 \pi)$ & Density $\left(\AA^{-3}\right)$ \\
\hline $\mathrm{Fe}_{90} \mathrm{Ni}_{10}$ & $\mathrm{Fe}_{0.9044} \mathrm{Ni}_{0.0956}$ & 0.9081 & 0.085 \\
$\mathrm{Fe}_{85} \mathrm{Ni}_{15}$ & $\mathrm{Fe}_{0.8562} \mathrm{Ni}_{0.1438}$ & 0.91583 & 0.08 \\
$\mathrm{Fe}_{85} \mathrm{Ni}_{5} \mathrm{~S}_{10}$ & $\mathrm{Fe}_{0.79311} \mathrm{Ni}_{0.04439} \mathrm{~S}_{0.1625}$ & 0.70788 & 0.075 \\
\hline \hline
\end{tabular}

$\rho=0.08 \AA^{-3}$, which corresponds to a value somewhat between that of the pure liquid $\mathrm{Fe}$ and liquid $\mathrm{Ni}$, was used.

In addition, all three samples present a low-angle scattering contribution as can easily be gauged from Fig. 1. For liquids such as ours, composed of atoms carrying welldefined magnetic moments (Fe, Ni), the simplest conceivable case is to consider scattering from randomly oriented (and uncorrelated) spins. This will give rise to a paramagnetic scattering contribution given by ${ }^{20}$

$$
\left(\frac{d \sigma}{d \Omega}\right)_{\text {para }}=\frac{2}{3} r_{0}^{2} S(S+1)[F(Q)]^{2},
$$

where $r_{0}=-0.5391 \times 10^{-12} \mathrm{~cm}$ is the classical electron radius times the neutron magnetic moment, $S=2$, with $3 d^{6}$ electrons, and $[F(Q)]$ stands for the average magnetic form factor (i.e., due to the spatial distribution of the unpaired atomic electrons). The latter has been previously determined ${ }^{21}$ for single-crystalline $\mathrm{Fe}_{0.66} \mathrm{Ni}_{0.34}$ at $300 \mathrm{~K}$ and found to be isotropic for the $Q$ range of interest here. It gives a value for the average localized moment of $1.48 \mu_{B}\left(1.80 \mu_{B}\right.$ for iron and $0.87 \mu_{B}$ for nickel), which is to be taken as an upper bound.

A comparison of the paramagnetic scattering contribution just referred to with the measured cross section for $\mathrm{Fe}_{0.90} \mathrm{Ni}_{0.10}$ is shown in Fig. 2. A glance to such graph shows that the low-angle data are confined to shorter wave numbers than those spanned by $F(Q)$, which may be taken as an indication of some degree of correlation between the magnetic moments. A first assessment of the nature of such small-angle signal was made by examining the shape of the riseup appearing for $Q$ values below $1 \AA^{-1}$ in terms $R(Q)^{-1}=(d \sigma / d \Omega)^{-1}$ versus $Q^{2}$. The rationale behind this is to identify whether such scattering arises from paramagnetic and/or critical fluctuations having a well-defined $Q$ dependence. For systems such as solid Fe-Ni Invar alloys ${ }^{22}$ or amorphous $\mathrm{Fe}$-based spin glasses ${ }^{23}$ within their paramagnetic phases, the observed magnetic scattering usually follows the Ornstein-Zernike (OZ) form

$$
R(Q)=\frac{A}{Q^{2}+\kappa^{2}},
$$

where $\kappa$ stands for the inverse of a correlation length and $A$ is an amplitude factor that can be calculated upon detailed knowledge of the exchange constant. Such a functional form would then yield straight-line plots for the inverse of the cross section versus $Q^{2}$. The inset of Fig. 2 shows that both samples follow the OZ prescription remarkably well. The slopes of such straight lines yield estimates for $A^{-1}$ and the intercepts give those for $A^{-1} \kappa^{2}$. The derived values for the latter parameters were $\kappa \approx 0.66 \AA^{-1}$ for both the binary alloy and the sulfur-containing samples. This yield real-space correlation radii of the order of $1.5 \AA$, indicating that some sort of spin correlations up to distances separating nearest neighbors (i.e., up to about $3 \AA$ ) still persist in the liquid state. On such grounds we approximate the small-angle contribution by the magnetic contribution to the cross section,

$$
\left(\frac{d \sigma}{d \Omega}\right)_{m a g}=\frac{2}{3} r_{0}^{2} \frac{1}{4}\left[F^{2}(Q)\right] R(Q),
$$

an example of which is also shown in Fig. 2.

To account for departures from the static approximation we have first calculated the terms entering the Placzek expansion of the cross section using the average atomic mass, temperature, incident neutron energy, and detector parameters as input. The calculated values for the $Q$-squared terms of the two binary alloys were of $2.3 \times 10^{-4} \AA^{2}$ and those for the fourth-power term were of $-2.6 \times 10^{-8} \AA^{4}$. The effect of the light element leads to an increase of the quadratic coefficient up to $2.0 \times 10^{-3} \AA^{2}$ while the fourth-order yields $-2.3 \times 10^{-8}$. Such a set of values is able to correct for most of the small drop in cross section at large angles. Somewhat improved estimates were derived from fits of the cross sec-

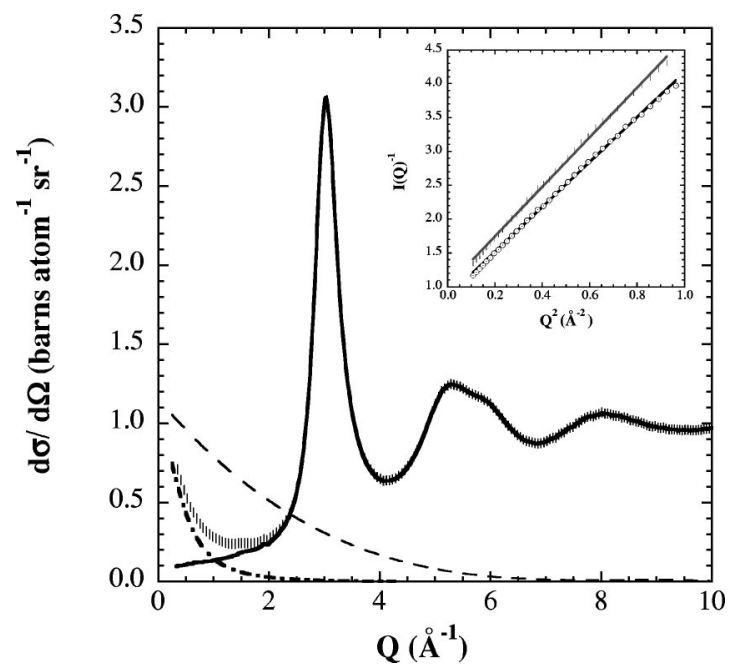

FIG. 2. A comparison of the measured $d \sigma / d \omega$ singledifferential scattering cross-section for molten $\mathrm{Fe}_{0.90} \mathrm{Ni}_{0.10}$ (vertical bars) including the magnetic contributions to low-angle scattering (dash-dots line) as well as the purely nuclear contribution (solid line). The long dashes represent the magnetic form-factor for $\mathrm{Fe}_{0.66} \mathrm{Ni}_{0.34}$ Invar alloy as measured at $300 \mathrm{~K}$ (see text). The inset depicts plots of the inverse of the measured intensity versus $\mathrm{Q}^{2}$ for $\mathrm{Fe}_{0.90} \mathrm{Ni}_{0.10}$ (line with vertical bars) and for $\mathrm{Fe}_{85} \mathrm{Ni}_{5} \mathrm{~S}_{10}$ (line with circles). 


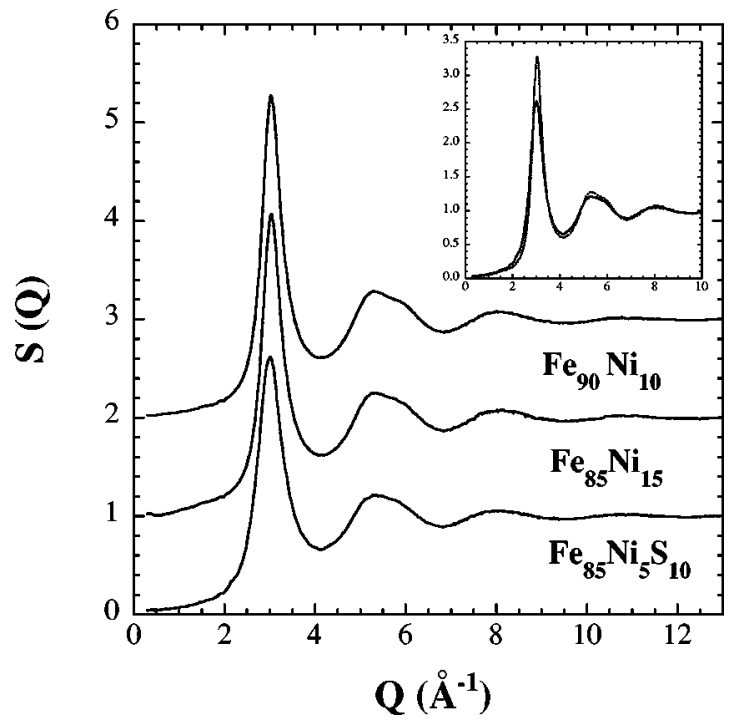

FIG. 3. Static structure factors for the three compositions as labeled. The curves have been shifted by one unit for the sake of clarity. The inset shows the structure factors for the $\mathrm{Fe}_{90} \mathrm{Ni}_{10}$ (dots) and the sulfur-containing sample (solid line) drawn onto a common scale.

tion to a polynomial with the first two even powers of $Q$, while keeping the maximum departure from the calculated quantities within $15 \%$.

The derived static structure factors obtained after subtraction of the magnetic component and normalized by the average squared coherent scattering lengths are shown in Fig. 3.

Extrapolation to $Q \rightarrow 0$ yields estimates for the compressibility limit $S(0)=\rho k_{B} T \kappa_{T}$ ), where $\kappa_{T}$ stands for the isothermal compressibility, of 0.026(4), 0.026(6), and 0.04(7) for the three samples, respectively. Such values can be are found to be consistent with estimates made from the macroscopic values of the sound velocity and densities for the three samples. ${ }^{7,8,24}$

The resulting $S(Q)-1$ were then transformed into real space to give $4 \pi \rho[G(r)-1]$ and a new set of improved estimates for the densities was then derived from its low- $r$ slope, which should follow $-4 \pi \rho r$. The process was done iteratively until no significant variations were detected and the final values are given in Table I.

\section{RESULTS}

Figure 3 shows the corrected structure factors for the three compositions. As expected, there are relatively small differences between spectra for both $\mathrm{Fe}_{x} \mathrm{Ni}_{1-x}$ samples. Maxima corresponding to the first peaks are located at $3.03 \AA^{-1}$ and their height varies from 3.07 for $\mathrm{Fe}_{90} \mathrm{Ni}_{10}$ to 2.90 for $\mathrm{Fe}_{85} \mathrm{Ni}_{15}$. In contrast, a strong decrease to 2.15 in the height of the first peak is observed for the sulfurcontaining sample that is accompanied by a small shift of $0.06 \AA^{-1}$ to lower wave vectors. Also, the latter sample shows a less marked structure in its second oscillation. The observation just refereed to indicates the presence of a less dense and less-structured liquid resulting from the action of the light element.

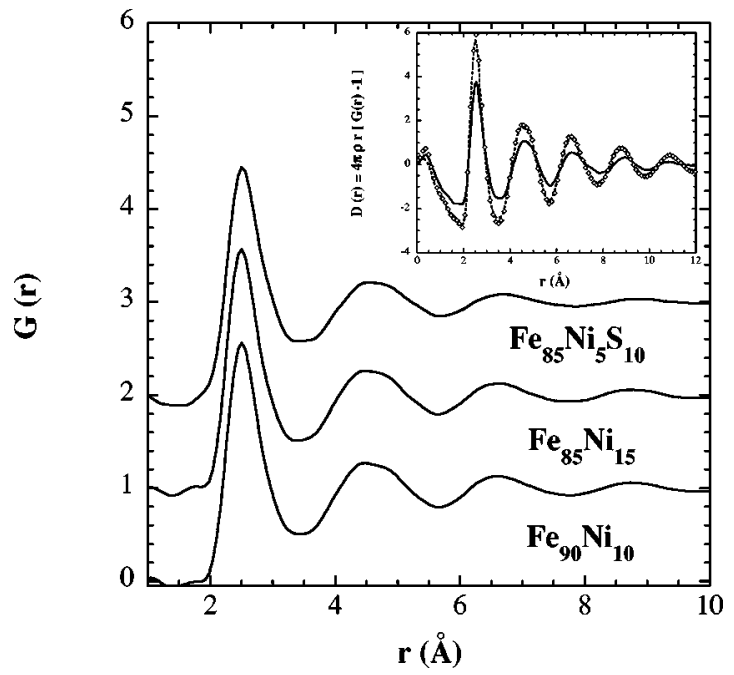

FIG. 4. Pair correlation functions for the three compositions as labeled. The curves are displaced by a factor of 1 for clarity. The inset compares the three $D(r) \propto r[G(r)-1]$ real-space correlation functions on the same scale. The thick line shows the function for the $\mathrm{Fe}_{85} \mathrm{Ni}_{5} \mathrm{~S}_{10}$ sample. Open lozenges show the correlation function for $\mathrm{Fe}_{90} \mathrm{Ni}_{10}$ and dashes that for $\mathrm{Fe}_{85} \mathrm{Ni}_{15}$.

The data corresponding to $G(r)$, which is the radial distribution function $g(r)$ weighted by the scattering lengths, are shown in Fig. 4. The graphs shown for the three samples depict the presence of significant oscillations that reveal rather long-ranged density correlations extending up to distances as long as $15 \AA$. The most remarkable feature here is the closeness of the phases of all three weighted pair distribution functions for distances smaller than $6 \AA$ as well as the notable differences exhibited by the $\mathrm{Fe}_{85} \mathrm{Ni}_{05} \mathrm{~S}_{10}$ samples with respect to the two binary alloys above such a cutoff value. In real numbers, the first peak of the three $G(r)$ 's is centered at $2.48 \AA$ with heights of $2.55,2.56$, and 2.41 for $\mathrm{Fe}_{90} \mathrm{Ni}_{10}, \mathrm{Fe}_{85} \mathrm{Ni}_{15}$, and $\mathrm{Fe}_{85} \mathrm{Ni}_{05} \mathrm{~S}_{10}$, respectively. Clear differences for the shape of the oscillations can be gauged from the inset of Fig. 4. The result is rather impressive since, as can be inferred from data shown in Table I as well as from the individual neutron coherent cross sections for $\mathrm{Fe}, \mathrm{Ni}$, and $\mathrm{S}$ that are $\sigma_{\mathrm{coh}}=11.22,13.3$, and $1.02 \mathrm{~b}$, respectively, most of the spectral features arise from $\mathrm{Fe}-\mathrm{Fe}$ correlations.

To make some quantitative estimate of the observed differences in the partial pair correlations, the functions $T(r)$ $=4 \pi \rho r^{2} G(r)$ were evaluated and are shown in Fig. 5. These quantities are defined as $T(r)=4 \pi r^{2} \sum_{i} \Sigma_{k} \rho_{i k} \bar{b}_{i} \bar{b}_{k} g_{i k}(r)$ where the latter stands for the partial pair correlation functions. There it is seen that the $T(r)$ distributions, the integral of which measures the number of particles within a volume element defined by a sphere with radius $r$, show significant differences between all the three samples. In fact, a rough estimate of the differences in microscopic densities is provided by integrals of such functions using as upper limits their local minima that are located at about $3.3 \AA$ and $5.6 \AA$ respectively. The particle numbers obtained are 13.7, 12.6, and 12.2, corresponding to the first peak of $\mathrm{Fe}_{90} \mathrm{Ni}_{10}$, $\mathrm{Fe}_{85} \mathrm{Ni}_{15}$, and $\mathrm{Fe}_{85} \mathrm{Ni}_{5} \mathrm{~S}_{10}$, respectively. The corresponding figures for integrals taken up to the second minima are 60.9, 


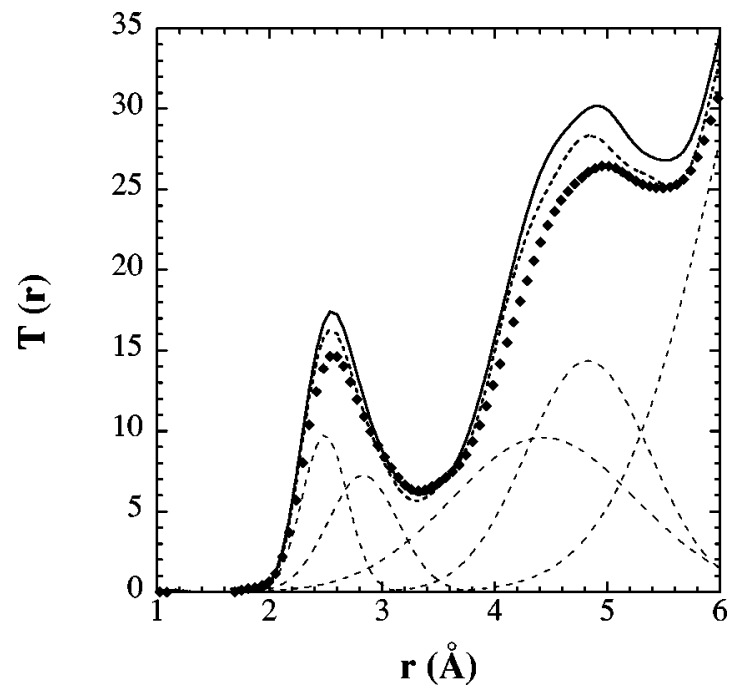

FIG. 5. T(r) for the three samples, solid line corresponds to the $\mathrm{Fe}_{90} \mathrm{Ni}_{10}$ sample, the dashes to $\mathrm{Fe}_{85} \mathrm{Ni}_{15}$ and filled symbols to $\mathrm{Fe}_{85} \mathrm{Ni}_{5} \mathrm{~S}_{10}$. The dashed lines display a decomposition of the latter curve in terms of a sum of five Gaussians.

59.0, and 53.8 respectively. An attempt to cast such differences in terms of coordination numbers was made by means of decomposition of $T(r)$ into a sum of four Gaussian functions plus an extra one included to take account of the tail of the higher-distance correlations. The procedure which does not provide unique estimates for the number of particles in the coordination spheres is here employed since our main interest is focused on differences between the three compositions rather than accurate estimates for individual samples.

The numerical values are given in Table II and their significance is addressed by comparison with simulation data.

An account made of the provisos referred to above the data shown in Table II suggests a transition from a liquid structure not far from that of the bcc structure of the parent Fe crystal that would correspond to values of 8 and 6 for the first two Gaussians and 8 and 24 for the former two, to a far less densely packed structure in $\mathrm{Fe}_{85} \mathrm{Ni}_{5} \mathrm{~S}_{10}$ where the numbers in its first coordination shell fall by about $22 \%$.

The figures given above can also be compared to those derived from $a b$ initio simulations on FeS. ${ }^{12}$ There it was found that the integral of the first peak of the radial distribution of FeS yields a coordination number of 10.8 that is to be compared with the sum of the first two rows of Table II. This would yield a value of 9.9 for the sulfur-containing samples while the sample having the largest iron content shows a higher coordination number of 12.7. Such trends are also reproduced in the above referred to simulation on $\mathrm{Fe}_{90} \mathrm{~S}_{10}{ }^{12}$ The relevant number for molten iron to compare such figures has been reported to be 13.8 (Ref. 12) rather close to 14 , which correponds to the bcc structure.

\section{DISCUSSION}

To understand with some more detail the observed trends in terms of particle configurations we have carried out a set of reverse Monte Carlo simulations (RMC) ${ }^{25}$ For such a purpose we have constructed simulation cells containing up to 20000 atoms with the proportions corresponding to the three compositions. ${ }^{26}$

Several starting configurations were chosen. First we chose a random configuration of all the atomic species with the only imposed constraint that the distance between all atoms cannot be less than $1.8 \AA$. A second set of simulations was carried out for the Fe-Ni-S sample where the initial configuration consisted in all sulfur atoms arranged in S-S pairs and S-S-S triplets. The geometric constraints on such units were those reported in Ref. 27 using a bond length of $2.08 \AA$ and a bond angle of $108.5^{\circ}$. Larger aggregates were not used since, as explained below, the optimization algorithm tends to tear apart the S-S pairs and, on the other hand, molten sulfur at $T \geqslant 1300 \mathrm{~K}$ is known to form small $\left[\mathrm{S}_{2}\right.$ to $\mathrm{S}_{5}$ (Ref. 27)] aggregates breaking any other larger chain or ring structure.

In all cases the initial configurations were later on modified following a Monte Carlo strategy ${ }^{25}$ so that the calculated structure functions were able to adjust to the experimental neutron diffraction patterns. An example of the quality of the fitted functions is shown in Fig. 6. From there one sees that the algorithm adequately reproduces the experimental observations in terms of particle configurations which give plausible atomic structures compatible with the observed $S(Q)$ [or $G(r)$ ].

From snapshots of the liquid structures as determined from RMC simulations some statistics about particle geometries were derived. Data for the calculated neutron-weighted $T(r)$ were found to adequately reproduce the figures given from integrals of such functions as referred to above. In addition we now have access to information on the $g_{i k}(r)$ and this provides a check on the estimated coordination numbers given in Table II. Because of the relative concentrations and scattering lengths of the three components, the most reliable results concern those positional correlations involving iron. A representative sample of those is shown in Fig. 7.

From Fig. 7 one gauges the following. First, the closest

TABLE II. Parameters defining the decomposition of the $T(r)$ distributions into sums of five Gaussians.

\begin{tabular}{lcccccc}
\hline \hline Gaussian & \multicolumn{2}{c}{$\mathrm{Fe}_{90} \mathrm{Ni}_{10}$} & \multicolumn{2}{c}{$\mathrm{Fe}_{85} \mathrm{Ni}_{15}$} & \multicolumn{2}{c}{$\mathrm{Fe}_{85} \mathrm{Ni}_{5} \mathrm{~S}_{10}$} \\
& Center $(\AA)$ & Coordination & Center $(\AA)$ & Coordination & Center $(\AA)$ & Coordination \\
\hline 1 & 2.46 & $6.6(3)$ & 2.44 & $5.4(4)$ & 2.45 & $4.9(4)$ \\
2 & 2.79 & $6.1(4)$ & 2.75 & $5.8(6)$ & 2.76 & $5.0(5)$ \\
3 & 4.18 & $16(1)$ & 4.1 & $18(1)$ & 4.11 & $19(2)$ \\
4 & 4.71 & $30(3)$ & 4.7 & $25(4)$ & 4.70 & $19(3)$ \\
\hline \hline
\end{tabular}




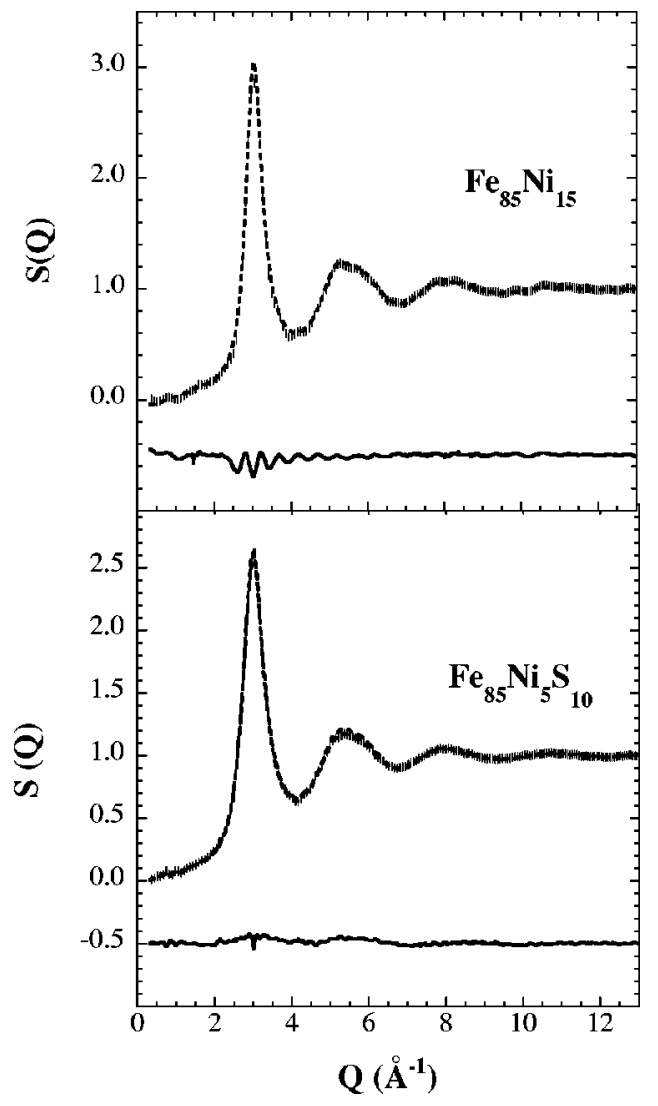

FIG. 6. Examples of approximations to the experimental structure factors derived from reverse Monte Carlo simulations. Experimental data are shown by dashed lines; the RMC fits as vertical bars and the residuals that are displaced by -0.5 units are shown by solid line.

Fe-Fe distance shifts to somewhat larger distances in passing from the binary alloy (peak at $2.46 \AA$ ) to the sulfurcontaining sample (peak at $2.5 \AA$ ) by an amount larger than that given in Table II. Also the peak intensity decreases, indicating a reduction in the coordination numbers covered by the first two Gaussians listed in Table II. Similar trends are also visible for the Fe-Ni correlations. Second, Fe-S correlations show a well-defined first peak at $2.36 \AA$ and subsidiary maxima at 4.4 and $6.6 \AA$. The value corresponding to the first peak compares favorably with that reported for crystalline FeS showing a troilite structure (a relative of the NiAs structure with $P \overline{6} 2 c$ space group). ${ }^{26}$ Its width, which becomes comparable to that of the other two pair correlations, indicates that $\mathrm{Fe}-\mathrm{S}$ short-ranged correlations last for short times only, thus suggesting that no stable Fe-S entities are formed. An estimate of the position of the first peak for Ni-S correlations (not shown) gives a value of $2.6 \AA$. The iron structure is to be compared with that reported for molten iron ${ }^{13}$ near melting where the main maximum of $g(r)$ is at about $2.56 \AA$, having a value of 2.54. From such a comparison we see that both binary and sulfur-containing alloys are closer packed at short range than molten iron and, in fact, the nearestneighbor $\mathrm{Fe}-\mathrm{Fe}$ correlations become now close to those shown by molten $\mathrm{Ni}$ (maxima of 2.36 at $2.46 \AA$ ).

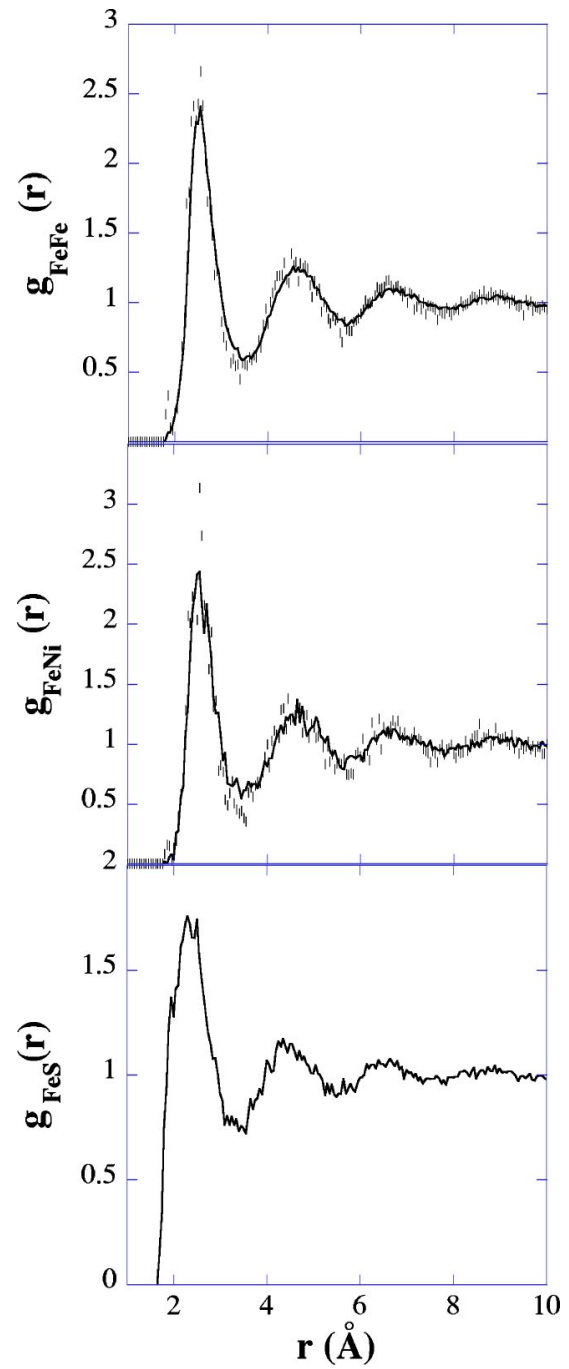

FIG. 7. Estimates for the radial distribution functions involving $\mathrm{Fe}$ atoms as derived from the RMC fits. Vertical dashes depict the functions for the $\mathrm{Fe}_{85} \mathrm{Ni}_{15}$ mixture and solid lines those for $\mathrm{Fe}_{85} \mathrm{Ni}_{5} \mathrm{~S}_{10}$.

A calculation of the distribution of bond angles subtended by three atoms within distances smaller than $3 \AA$ from the origin was carried out. The main results are shown in Fig. 8 which compares an average angle subtended by three atoms all of which are $\mathrm{Ni}$ or $\mathrm{Fe}$ with that corresponding to a triplet where one of the atoms corresponds to sulfur. The figure just referred to also serves to compare the angles subtended by the central atom and its nearest neighbors within a bcc lattice with the calculated average distribution for triplets containing $\mathrm{Fe}$ or $\mathrm{Ni}$. The picture that emerges from here portrays the local geometry of $\mathrm{Fe}$ or $\mathrm{Ni}$ within the alloys as close to that of the native bcc structure of low-temperature Fe. On the other hand, as also seen from Fig. 8 the introduction of sulfur leads to a wider distribution of bond angles, making the local structure to further depart from the bcc reference.

No significant S-S correlations were found in $\mathrm{Fe}_{85} \mathrm{Ni}_{5} \mathrm{~S}_{10}$ after extensive searches over many particle configurations and initial conditions. In fact, our present data and the subsequent analysis of the basis of the RMC results are consis- 


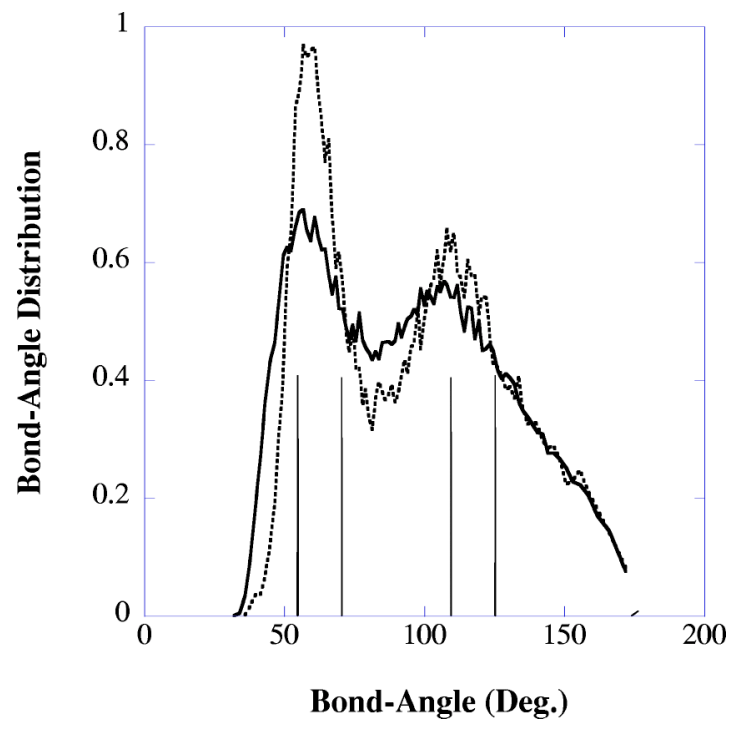

FIG. 8. Distribution of bond angles as calculated from particle configurations derived from RMC fits. The dots display an average of bond angles for triplets of atoms formed by Fe or Ni. The solid line depicts the corresponding distribution for the sulfur-containing sample for triplets containing least one sulfur atom. The vertical bars show the angles for a bcc lattice.

tent with a fully randomized distribution of $\mathrm{S}$ atoms over the whole metallic Fe-Ni matrix. Moreover, no clear signature of a well defined S-S bond is seen either as a molecular form factor contributing to $S(Q)$ at large $Q$ values or as an identifiable peak appearing at about $2 \AA$ in the static correlation function. This finding is in apparent contrast with the conjecture put forward by Nasch et al. ${ }^{7}$ of the formation of long aggregates of "molecular" sulfur that break down with increasing temperature as a main mechanism to explain the ultrasound data. Our data, while in contrast with arguments given by Ref. 7 are in line with first-principles calculations reported in Ref. 12, where no tendency to polymerization of sulfur was found, and the same applies to calculations reported by Tse and King et $a .^{27}$

The simplest liquid dynamics quantity that can be calculated from knowledge of the static structure factors $S(Q)$ is the square root of the spectral second frequency moment defined as

$$
\left\langle\omega_{0}^{2}\right\rangle^{1 / 2}=\sqrt{\frac{\int \mathrm{d} \omega \omega^{2} S(Q, \omega)}{S(Q)}}=\sqrt{\frac{k_{B} T \hbar^{2} Q^{2}}{M S(Q)}},
$$

where $S(Q, \omega)$ stands for the dynamic structure factor and $M$ is the average atomic mass. This quantity has the low- $Q$ limit of $\lim _{Q \rightarrow 0}\left\langle\omega_{0}^{2}\right\rangle^{1 / 2}=Q c_{T}$, where $c_{T}$ stands for the isothermal sound velocity. Figure 9 displays the wave-vector dependence of $\left\langle\omega_{0}^{2}\right\rangle^{1 / 2}$ for a binary melt as well as the sulfurcontaining sample. The hydrodynamic dispersion laws are calculated from values of the isothermal sound velocities derived from the low-Q limit of $S(Q)$. These values are $3661 \mathrm{~m} \mathrm{~s}^{-1}$ for $\mathrm{Fe}_{90} \mathrm{Ni}_{10}, 3242 \mathrm{~m} \mathrm{~s}^{-1}$ for $\mathrm{Fe}_{85} \mathrm{Ni}_{15}$, and $2741 \mathrm{~m} \mathrm{~s}^{-1}$ for $\mathrm{Fe}_{0.85} \mathrm{Ni}_{0.05} \mathrm{~S}_{0.15}$, respectively, and are to be

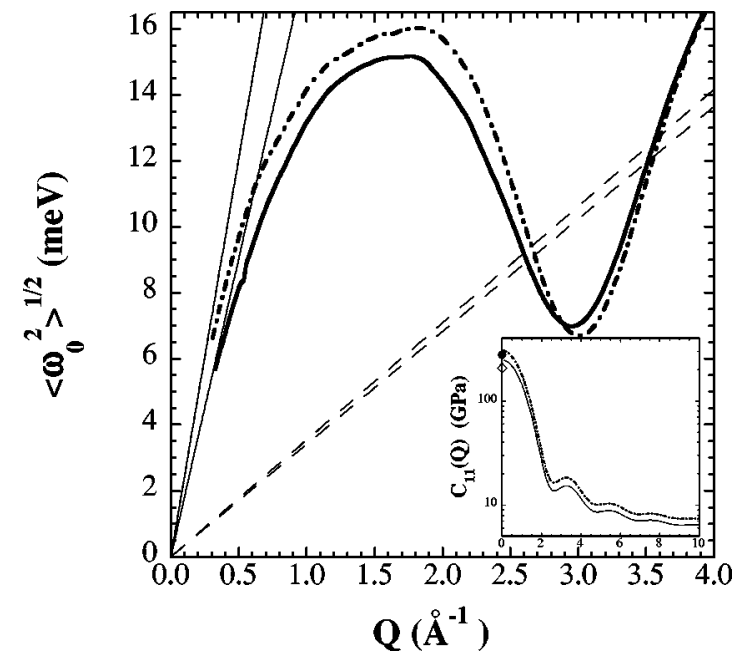

FIG. 9. Reduced spectral second frequency moments for $\mathrm{Fe}_{85} \mathrm{Ni}_{5} \mathrm{~S}_{10}$ (solid line) and $\mathrm{Fe}_{90} \mathrm{Ni}_{10}$ (thick dashes). The straight lines correspond to hydrodynamic dispersion laws $\omega=c Q$, calculated with the isothermal sound velocities (Ref. 24). The thin dashed lines represent the values of the ideal-gas limits $\left\langle\omega_{\text {id }}^{2}\right\rangle^{1 / 2}$ $=\sqrt{Q^{2} k_{B} T / M}$, where $M$ stands for the mean atomic mass. The inset depicts a comparison of the wave-vector-dependent elastic moduli $C_{11}(Q)$ for both samples. Symbols at $Q=0$ stand for the corresponding quantity for bcc iron at $300 \mathrm{~K}$ (solid symbol) and an $\mathrm{FeNi}_{31}$ alloy at $1373.5 \mathrm{~K}$ (open symbol). See Ref. 34 .

compared to those for the adiabatic sound velocity as measured by ultrasound $c_{v}(T)=\sqrt{\gamma c_{T}^{2}}$. The value for the $\mathrm{Fe}_{90} \mathrm{Ni}_{10}$ sample is $4244 \mathrm{~m} \mathrm{~s}^{-1}$ using a Grüneisen value of 1.344 (Ref. 24). Such a value is somewhat below that of the $4400 \mathrm{~m} \mathrm{~s}^{-1}$ figure quoted for molten Fe above melting, ${ }^{8}$ but above that of $3820 \mathrm{~m} \mathrm{~s}^{-1}$ also quoted at melting by Nasch et $a .^{24}$ Our estimate for the sulfur-containing sample compares to that reported by Nasch et al. ${ }^{7}$ if a value of about 1.36 is used for the Grüneisen parameter $\gamma$.

In both cases depicted in Fig. 9 the hydrodynamic regime of $\left\langle\omega_{0}^{2}\right\rangle^{1 / 2}$ is well approached from below. Also, at large momentum transfers the oscillations follow the corresponding ideal-gas limits given by the particle thermal velocities only. The approach to the $\omega=c Q$ hydrodynamic dispersion from below contrasts with the behavior of the physical excitation frequencies which in the molten state of $3 d$ metals such as molten Ni (Ref. 28) approaches the hydrodynamic dispersion from above, a phenomenon known as positive dispersion. The latter is found to be ubiquitous in molten metallic alloys $^{29}$ or in pure metals ${ }^{30}$ and is known to be crucial to enable the decay of phonon excitations. ${ }^{31}$

An estimate of the generalized high-frequency elastic constant $C_{11}(Q)$ may be obtained from the relationship ${ }^{32}$

$$
C_{11}(Q)=\frac{M \rho \omega_{l}^{2}}{Q^{2}},
$$

where the term in the numerator stands for the mass density times a quantity related to the fourth-frequency moment of $S(Q, \omega)$. Because of the lack of detailed knowledge of the latter quantity, an approximation for $\omega_{l}$ is given 
by $\omega_{l}^{2} \doteq\left(3 Q^{2} k_{B} T / M\right)+\omega_{E}^{2}\left(1-3 \sin Q_{\text {red }} / Q_{\text {red }}-6 \cos Q_{\text {red }} /\right.$ $Q_{\text {red }}^{2}+6 \sin Q_{\text {red }} / Q_{\text {red }}^{3}$ ) with $Q_{\text {red }}=Q R_{\max }$ where $R_{\max }$ stands for the position of the main peak of $g(r)$. Estimates for the "Einstein frequencies" $\omega_{E}$ are again made from established correlations based upon the Lindemann melting criterion. ${ }^{33}$ The low- $Q$ limit of $C_{11}(Q)$ yields the sum $B+4 G / 3$ of bulk and shear modulus and represents the instantaneous, solidlike response of the collective oscillation. This enables to establish a comparison with elastic data for the solid and this is done in the inset of Fig. 9 where the wave-vectordependent quantities are compare with macroscopic data for pure bcc iron as well as for some some Invar alloys. ${ }^{34}$ As seen there the high-frequency response of molten $\mathrm{Fe}_{90} \mathrm{Ni}_{10}$ becomes at short interaction times comparable to that of bcc iron and the same applies to that of the sulfur-containing sample.

\section{CONCLUSION}

As a main conclusion, the data quoted above show that relevant structural details as well as some insight into the dynamics of alloys suspected to be present within the Earth's outer core can be derived by means of neutron diffraction. This opens the way for future experiments using isotopic substitution in order to derive firsthand experimental infor- mation on the partial pair correlation functions.

The data at hand suggest the presence of rather welldefined Fe-S bonds possibly of a covalent character as pointed in Ref. 12 although its binding strength does not seem to be high enough to consider such units as " "molecules."

From the information here derived we also attest to the dramatic effect brought forward by the light element into the isothermal Young modulus of these samples. Indeed, from the figures quoted above concerning the sound velocity and densities given in Table I one sees that the reduction in value of this elastic property for the sulfur-containing sample with respect to the two binary alloys amounts to $54 \%$ for $\mathrm{Fe}_{90} \mathrm{Ni}_{10}$ and $38 \%$ for $\mathrm{Fe}_{85} \mathrm{Ni}_{15}$. Detailed microscopic studies on how the elastic properties of these melts are so dramatically perturbed by the addition of the light alloying element are now underway.

\section{ACKNOWLEDGMENTS}

We thank the Institut Laue Langevin for neutron beam time. We are grateful to Pierre Palleau and to Dr. J. Campo of ILL for his technical support during the diffraction experiments, and Carlos Ayala of Centro Atómico Bariloche for the sample preparation.
*Also at Instituto de Estructura de la Materia, C.S.I.C., Serrano 123, E-28006 Madrid, Spain.

${ }^{1}$ F. Birch, J. Geophys. Res. 69, 4377 (1964).

${ }^{2}$ J.P. Poirier, Phys. Earth Planet. Inter. 85, 319 (1994).

${ }^{3}$ G.K. Schubert, K. Zhang, M.G. Kivelson, and J.D. Anderson, Nature (London) 384, 544 (1996).

${ }^{4}$ T. J. Ahrens, in Shock Compression of Condensed Matter-1995, edited by S. C. Schmidt and W. C. Tao, AIP Conf. Proc. 370, 3 (1996).

${ }^{5}$ C. Sanloup, F. Guyot, P. Gillet, and Y. Fei, J. Geophys. Res., [Solid Earth] 107, 2272 (2002).

${ }^{6}$ J.D. Anderson, W.L. Sjogren, and G. Schubert, Science 272, 709 (1996)

${ }^{7}$ P.M. Nasch, M.H. Manghnani, and R.A. Secco, Science 277, 219 (1997).

${ }^{8}$ T. Iida and R. I. L. Guthrie, The Physical Properties of Liquid Metals (Oxford University Press, New York, 1993), Chaps. 4 and 6. See also R.S. Hixson, M.A. Winkler, and M.L. Hodgdon, Phys. Rev. B 42, 6485 (1990).

${ }^{9}$ H. Vollstädt, U. Seiplod, and A. Kraft, Phys. Earth Planet. Inter. 22, 267 (1980); Y. Kita, Trans. ISIJ (Japan) 22, 571 (1982).

${ }^{10}$ D. Alfè, G.D. Price, and M.J. Gillan, Phys. Rev. B 65, 165118 (2002).

${ }^{11}$ D. Alfè, G. Kresse, and M.J. Gillan, Phys. Rev. B 61, 132 (2000).

${ }^{12}$ D. Alfè and M.J. Gillan, Phys. Rev. B 58, 8248 (1998), and referenecs therein.

${ }^{13}$ Y. Waseda, The Structure of Noncrystalline Materials (McGrawHill, New York, 1980).

${ }^{14}$ R. Winter, P.A. Egelstaff, W.C. Pilgrim, and W.S. Howells, J. Phys.: Condens. Matter 2, SA215 (1990); R. Bellissent, L. Descotes, F. Boué, and P. Pfeuty, Phys. Rev. B 41, 2135 (1990); J.S. Tse and D.D. Klug, ibid. 59, 34 (1999).
${ }^{15}$ H.E. Fischer, G.J. Cuello, P. Palleau, D. Feltin, A.C. Barnes, Y.S. Badyal, and J.M. Simonson, Appl. Phys. A: Mater. Sci. Process. 74, S160 (2002).

${ }^{16}$ J. Blétry, P. Tavernière, C. Senillou, P. Desré, M. Maret, and P. Chieux, Rev. Phys. Appl. 19, 725 (1984).

${ }^{17}$ H. Bertagnolli, P. Chieux, and M.D. Zeidler, Mol. Phys. 32, 759 (1976).

${ }^{18}$ J.F. Jal, C. Mathieu, P. Chieux, and J. Dupuy, Philos. Mag. B 62, 351 (1990).

${ }^{19}$ M. A. Howe, R. L. McGreevy, and P. Zetterström, Computer code CORRECT, Correction program for neutron diffraction data, NFL Studsvik, 1996.

${ }^{20}$ W. Gavin Williams, Polarized Neutrons (Clarendon Press, Oxford, 1988), p. 53.

${ }^{21}$ Y. Ito, J. Akimitsu, M. Matsui, and S. Chikazumi, J. Magn. Magn. Mater. 10, 194 (1979).

${ }^{22}$ See for instance S.V. Grigoriev, S.A. Klimko,. W.H. Kraan, A.I. Okorokov, M.Th. Rekveldt, and V.V. Runov, Phys. Rev. B 64, 094426 (2001); S.V. Grigoriev, S.V. Maleyev, A.I. Okorokov, and V.V. Runov, ibid. 58, 3206 (1998); K. Tajima, P. Böni, G. Shirane, Y. Ishikawa, and M. Koghi, ibid. 35, 274 (1987).

${ }^{23}$ G. Aeppli, S.M. Shapiro, R.J. Birgenau, and H.S. Chen, Phys. Rev. B 28, 5160 (1983).

${ }^{24}$ Sound velocity for the sulfur-containing sample taken from Ref. 7. For the binary alloys use is made of the empirical equation based upon the Lindemann melting criterion, $c$ $=18 \beta \sqrt{\left(R T_{m} / M_{\mathrm{av}}\right)}$ taken from, Ref. 8 p. 98 . Here $\beta=0.48$ stands for a correction factor and $M_{\mathrm{av}}$ is the average atomic mass. Experimental values for molten iron are given by P.M. Nasch, M.H. Manghnani, and R.A. Secco, J. Geophys. Res. 99, 4285 (1994). An equation of state for liquid iron that includes estimates for the thermodynamic Grüneisen parameters is given 
by W.W. Anderson and T.J. Ahrens, J. Geophys. Res. 99, 4273 (1994).

${ }^{25}$ R.L. McGreevy and L. Pusztai, Mol. Simul. 1, 359-367 (1998).

${ }^{26}$ H.E. King, Jr. and C.T. Prewitt, Acta Crystallogr., Sect. B: Struct. Crystallogr. Cryst. Chem. 38, 1877 (1982).

${ }^{27}$ J.S. Tse and D.D. Klug, Phys. Rev. B 59, 34 (1999).

${ }^{28}$ F.J. Bermejo, M.L. Saboungi, D.L. Price, M. Alvarez, B. Roessli, C. Cabrillo, and A. Ivanov, Phys. Rev. Lett. 85, 106 (2000).

${ }^{29}$ M. Alvarez, F.J. Bermejo, P. Verkerk, and B. Roessli, Phys. Rev. Lett. 80, 2141 (1998); R. Fernandez-Perea, M. Alvarez, F.J. Bermejo, P. Verkerk, B. Roessli, and E. Enciso, Phys. Rev. E 58, 4568 (1998).

${ }^{30}$ C. Cabrillo, F.J. Bermejo, M. Alvarez, P. Verkerk, A. Maira-Vidal, S.M. Bennington, and D. Martin, Phys. Rev. Lett. 89, 075508 (2002); see also Ref. 28.
${ }^{31}$ F.J. Bermejo, B. Fåk, S.M. Benninton, R. Fernandez-Perea, C. Cabrillo, J. Davidowski, M.T. Fernandez-Diaz, and P. Verkerk, Phys. Rev. B 60, 15154 (1999); H.J. Maris, Rev. Mod. Phys. 49, 341 (1977).

${ }^{32}$ S. W. Lovesey, Neutron Scattering from Condensed Matter (Clarendon Press, Oxford, 1984) p. 181.

${ }^{33}$ See Eq. (5.25) in Ref. 8, p. 126 and also p. 100. The calculated values yield $25.1 \mathrm{meV}$ for the binary alloy and $24.8 \mathrm{meV}$ for the sulfur-containing sample.

${ }^{34}$ Data corresponding to bcc iron at $300 \mathrm{~K}$ are taken from S. Klotz and M. Braden, Phys. Rev. Lett. 85, 3209 (2000). Also for fcc and hcp data for iron are given in P. Söderlind, J.A. Moriarty, and J.M. Wills, Phys. Rev. B 53, 14063 (1996). Elastic data for $\mathrm{FeNi}_{31.5}$ are taken from $\mathrm{Ph}$. Renaud and S.G. Steinemann, Physica B 161, 75 (1989). 